J. Phys. IV France 127 (2005) 91-98

(C) EDP Sciences, Les Ulis

DOI: $10.1051 /$ jp4:2005127014

\title{
Contrôle de la génération d'harmoniques d'ordres élevés par modulation de l'ellipticité du fondamental
}

\author{
A. Zaïr ${ }^{1}$, I.J. Sola ${ }^{1}$, R. López-Martens ${ }^{2}$, P. Johnsson², E. Cormier ${ }^{1}$, \\ K. Varjù ${ }^{2}$, J. Mauritsson ${ }^{2}$, D. Descamps ${ }^{1}$, V. Strelkov ${ }^{1}$, A. I'Huillier ${ }^{2}$, \\ E. Mével ${ }^{1}$ et $\mathrm{E}$. Constant ${ }^{1}$ \\ ${ }^{1}$ CELIA (Centre Lasers Intenses et Applications), UMR 5107 du CNRS, \\ Université Bordeaux I, 351 cours de la Libération, 33405 Talence Cedex, France \\ e-mail : zair@celia.u-bordeaux1.fr \\ ${ }^{2}$ LLC (Lund Laser Center), Departement of Physics, Lund Institute of Technology, \\ PO Box 118, 22100 Lund, Sweden
}

\begin{abstract}
Résumé. Nous présentons les résultats de deux campagnes expérimentales, étudiant le confinement temporel de la génération d'harmoniques d'ordres élevés par modulation de l'ellipticité du champ fondamental. Une première expérience réalisée au LLC (Lund Laser Center) valide cette technique de «porte d'ellipticité » dans le régime femtoseconde et nous permet par une mesure temporelle directe de comparer la durée de l'émission XUV avec la durée calculée de la porte d'ellipticité. Une seconde expérience réalisée au CELIA met en évidence un élargissement spectral des harmoniques jusqu'à l'obtention d'un quasi-continuum. Ce quasicontinuum est théoriquement consistant avec l'émission d'une impulsion ou de deux impulsions attosecondes selon la phase absolue du champ fondamental.
\end{abstract}

\section{INTRODUCTION}

Durant les dernières décades, le développement de sources lasers femtosecondes a permis l'étude de processus ultrarapides dans la matière. Néanmoins, les processus survenant à l'échelle subfemtoseconde (relaxations électroniques) demeuraient inaccessibles. Il a été démontré récemment [1,2] que les harmoniques d'ordres élevés produites par interaction non linéaire entre un gaz atomique et une impulsion laser IR courte et intense étaient émises sous forme d'un train d'impulsions XUV attosecondes $\left(10^{-18} \mathrm{~s}\right)$. L'objectif est désormais de produire une impulsion attoseconde isolée qui, dans le cadre d'une expérience pompe-sonde, permettrait d'observer la dynamique des électrons dans des systèmes atomiques, moléculaires ou solides. Un défi expérimental actuel consiste donc à confiner temporellement l'émission harmonique afin de produire une impulsion attoseconde unique. On peut comprendre comment la génération d'harmoniques d'ordres élevés (HHG) peut produire des impulsions attosecondes en examinant son spectre. En effet, le spectre se présente sous la forme d'un peigne de fréquences harmoniques multiples impaires de la fréquence infra-rouge (IR). Si les harmoniques sont émises en phase, ce spectre harmonique XUV correspond temporellement à un train d'impulsions attosecondes émises tous les demi-cycles optique IR $[3,4,5]$. La génération d'harmoniques par un atome unique peut être décrite sur une période optique laser par un modèle semi-classique en trois étapes: l'ionisation atomique par effet tunnel, l'accélération de l'électron émis dans le continuum par le champ laser et la recombinaison radiative de cet électron avec son ion parent. C'est lors de cette recombinaison radiative qu'un photon harmonique d'énergie $\mathrm{E}_{\mathrm{H}}=\mathrm{I}_{\mathrm{p}}+\mathrm{E}_{\mathrm{c}}$ est émis ( $\mathrm{I}_{\mathrm{p}}$ : Potentiel d'ionisation de l'atome de gaz et $\mathrm{E}_{\mathrm{c}}$ : Energie cinétique au moment de la collision) [3,4]. Cette dernière étape ne peut avoir lieu que si la polarisation du laser permet un retour de l'électron au voisinage de l'ion. L'efficacité harmonique est donc maximale pour une polarisation IR linéaire (ellipticité $\varepsilon=0$ ) et décroît typiquement de $50 \%$ pour une ellipticité de l'ordre de $15 \%$. Une méthode pour produire une seule impulsion attoseconde dans le train consiste alors à créer une porte de polarisation quasi-linéaire $(\varepsilon<15 \%)$ dans l'impulsion laser IR d'une durée inférieure à un demi-cycle optique. Pour cela nous avons mis en oeuvre au 
CELIA une technique [3] permettant de moduler temporellement l'ellipticité du fondamental et de créer cette «porte temporelle d'ellipticité». Les campagnes d'expériences réalisées ont eu pour objectif de mettre en évidence, d'une part, le confinement temporel de l'émission harmonique XUV, et d'autre part, l'élargissement spectral des harmoniques lorsque l'on fait varier la durée de la porte temporelle d'ellipticité. Dans cet article, nous allons tout d'abord décrire la technique de porte d'ellipticité utilisée. Nous présenterons ensuite deux résultats expérimentaux qui nous ont permis de mesurer la durée de l'émission XUV pour trois configurations particulières de la porte d'ellipticité et de montrer la possibilité d'obtenir via cette technique un quasi-continuum spectral consistant avec la production d'une ou deux impulsions attosecondes. Enfin nous conclurons sur les perspectives envisagées.

\section{LA PORTE D'ELLIPTICITÉ}

Pour créer cette fenêtre temporelle dans l'impulsion fondamentale, où la polarisation IR est quasilinéaire $(\varepsilon<15 \%)$, il faut faire évoluer temporellement l'ellipticité du fondamental pendant la durée de l'impulsion. Afin d'optimiser l'efficacité de génération des harmoniques, la polarisation linéaire doit être produite au maximum d'intensité laser. Pour cela nous utilisons un jeu de deux lames de quartz quart d'onde $(\lambda / 4)$ respectivement d'ordre multiple et d'ordre zéro (figure 1.1).

Les axes neutres de la première lame sont fixés à $\alpha=45^{\circ}$ de la polarisation laser incidente. De cette façon, l'impulsion laser incidente de durée $\tau$ est séparée en deux composantes ordinaire (o) et extraordinaire (e) décalées temporellement l'une par rapport à l'autre de $\delta \approx \tau$. Le profil temporel ainsi obtenu a une durée de $2 \tau(\mathrm{FWHM})$ et compte tenu du déphasage induit par la lame $((2 \mathrm{~m}+1) \times \pi / 2$ avec $\mathrm{m}$ entier), il présente une polarisation circulaire au centre de l'impulsion et une polarisation linéaire dans les ailes de l'impulsion. La seconde lame, dont les axes neutres sont orientés selon un angle variable $\beta$ par rapport à la direction de polarisation du champ laser incident, permet de modifier l'état de polarisation de cette impulsion tout en conservant le même profil temporel $(2 \tau)$.

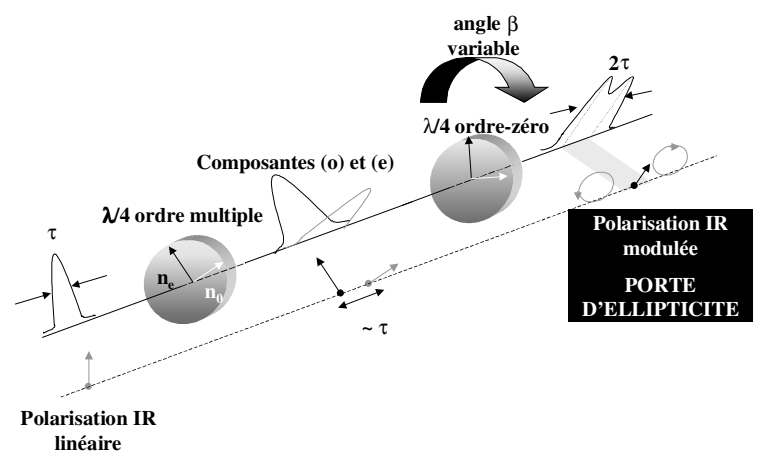

Figure 1.1. Dispositif expérimental de la porte d'ellipticité.

Ainsi lorsque $\beta=0^{\circ}$, la polarisation circulaire est transformée en linéaire et vice versa. On définit alors la «porte d'ellipticité» comme étant la fenêtre temporelle pour laquelle l'ellipticité $\varepsilon$ est inférieure à $15 \%$. En faisant varier $\beta\left(\beta \neq 0^{\circ}\right)$, on change l'ellipticité dans les ailes de l'impulsion mais la polarisation reste linéaire au centre. En modifiant l'orientation des axes de la seconde lame ( $\beta$ variable) on peut ainsi faire varier continûment la durée de cette porte ce qui permet en principe de contrôler le nombre d'impulsions attosecondes dans le train. Notons trois configurations particulières du dispositif: La configuration «porte étroite», obtenue lorsque $\beta=0^{\circ}$, correspond à une durée de la porte d'ellipticité minimale. La configuration «porte large», obtenue lorsque $\beta=45^{\circ}$, correspond à une porte de durée « infinie », c'est à dire que le champ laser est alors linéairement polarisé pendant toute la durée de l'impulsion, mais l'orientation de cette polarisation tourne de $90^{\circ}$ entre le début et la fin de l'impulsion IR. Lorsque $\beta=\alpha=0^{\circ}$, on définit 
la configuration «sans porte» qui correspond à la propagation de l'impulsion incidente selon un des axes neutres des deux lames n'induisant ainsi aucun changement ni dans l'état de polarisation (polarisation linéaire de direction constante) de l'impulsion fondamentale ni dans le profil temporel initial (durée $\tau$ ). Notons que la dispersion induite par les deux lames est pré-compensée en amont du dispositif par un réglage du compresseur de la chaîne laser. La figure (1.2) représente en configuration « porte étroite » la forme du profil temporel et l'évolution de l'ellipticité IR pour une impulsion incidente de durée $\tau=40 \mathrm{fs}$ et un délai $\delta=30 \mathrm{fs}$ introduit par la lame $\lambda / 4$ d'ordre multiple.

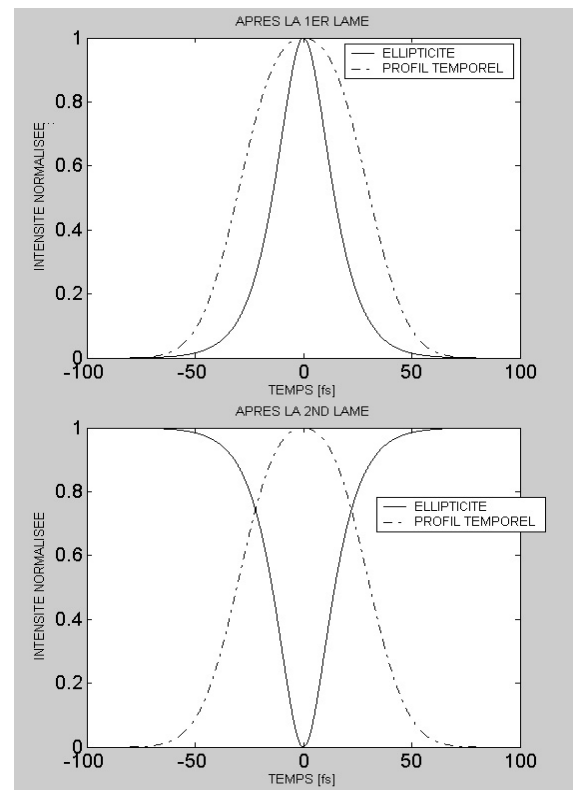

Figure 1.2. Modulation temporelle de l'ellipticité d'une impulsion IR de $\tau=40 \mathrm{fs}$ avec un délai $\delta=30 \mathrm{fs}$.

Après la première lame, l'ellipticité $\varepsilon(\mathrm{t})$ (courbe continue) est maximale au centre du profil $(\varepsilon=1)$ et décroît de part et d'autre. Après la seconde lame, on peut voir que le profil d'intensité (courbe pointillée) est conservé mais que l'ellipticité est « inversée». En configuration «porte étroite» la durée de la porte est de 11,6 fs pour un critère d'ellipticité de $\varepsilon(\mathrm{t}) \leq 15 \%$. La durée $\tau_{\text {gate }}$ de la porte d'ellipticité peut être calculée de la façon suivante :

$$
\tau_{\text {gate }}=\frac{\varepsilon \cdot \tau_{I R}^{2}}{\ln 2 \delta \cos (2 . \beta)}
$$

où $\varepsilon$ désigne l'ellipticité $(15 \%)$, $\tau_{\text {IR }}$ désigne la durée de l'impulsion IR incidente, $\delta$ le délai entre les composante ordinaire (o) et extraordinaire (e) après la lame $\lambda / 4$ d'ordre multiple et $\beta$ l'angle de la seconde lame $\lambda / 4$ [6]. Avec une impulsion IR de départ de $\tau_{\mathrm{IR}}=9 \mathrm{fs}$ et un délai de $\delta=15 \mathrm{fs}$, la durée de la porte d'ellipticité ( $\tau_{\text {gate }}=1,16 \mathrm{fs}$ ) est alors inférieure à un demi-cycle optique IR (demi-cycle optique à $800 \mathrm{~nm}$ $=1,33 \mathrm{fs}$ ). On assure ainsi théoriquement l'émission d'une à deux impulsions attosecondes suivant la phase absolue du champ laser [6]. La technique de « porte d'ellipticité » est simple à mettre en œuvre expérimentalement et possède l'avantage de confiner le processus de génération d'harmonique dans une gamme spectrale très large compatible à priori avec l'obtention d'impulsions approchant les 100 as. Une autre technique permettant d'isoler une seule impulsion attoseconde dans le train consiste à générer des harmoniques d'ordres élevés avec des impulsions lasers de 5 fs et à sélectionner spectralement les harmoniques les plus hautes qui sont uniquement produites pendant une seule demi-période optique prés du pic de l'impulsion [7]. La sélection spectrale inhérente à cette autre méthode impose une limitation de principe à la durée de l'impulsion attoseconde. 


\section{MESURE TEMPORELLE DU CONFINEMENT HHG PAR CORRÉLATION CROISÉE (IR/XUV) RÉSOLUE EN TEMPS}

Dans le cadre du réseau européen «ATTO», nous avons eu l'occasion de collaborer avec le groupe harmonique du Lund Laser Center (LLC). Une première collaboration en 2002 nous a permis de mesurer temporellement le confinement harmonique par une expérience de corrélation croisée entre une impulsion IR de 12 fs et une impulsion harmonique XUV produite par une impulsion IR de 35 fs d'ellipticité temporellement modulée. Cette expérience nous a permis de mettre en évidence un contrôle clair de la durée d'émission XUV en fonction de la durée de la porte d'ellipticité et ainsi de valider la technique de modulation temporelle d'ellipticité IR [8]. Cependant, compte tenue de la durée de la sonde IR (12 fs) et de la géométrie non colinéaire du dispositif expérimental, nous n'avons pas obtenu une résolution temporelle permettant de relier directement la mesure du confinement HHG à la durée de la porte d'ellipticité. Dans ce but, nous nous sommes donc proposé de réaliser cette expérience avec une sonde plus courte (9fs) et en géométrie colinéaire.

\subsection{Dispositif expérimental}

Le laser Titane:Saphir du LLC délivre des impulsions IR $\left(\lambda_{\mathrm{IR}}=815 \mathrm{~nm}\right)$ de $40 \mathrm{fs}$ à une cadence de $1 \mathrm{kHz}$. En sortie de la chaîne laser nous disposons de deux lignes de $1 \mathrm{~mJ}$ chacune. La première ligne est utilisée pour produire la sonde IR ultra-brève par une technique de post-compression dans une fibre creuse remplie d' argon $\left(\mathrm{P}_{\mathrm{Ar}}=300 \mathrm{mbar}\right)[9,10]$. En sortie de fibre et après compensation de la dispersion par une succession de réflexion sur des miroirs à dérive de fréquence négative, nous disposons d'impulsions IR de 9 fs et d'une énergie totale de $500 \mu \mathrm{J}$. Sur la seconde ligne, la polarisation des impulsions IR de durée $40 \mathrm{fs}$ est modulée à l'aide des lames quart d'onde ( $\delta=30 \mathrm{fs}$ ). Ces impulsions sont directement focalisées par un miroir sphérique ( $\mathrm{f}=+500 \mathrm{~mm}$ ) dans une cellule de $3 \mathrm{~mm}$ de long remplie d'argon $\left(\mathrm{P}_{\mathrm{Ar}}=30 \mathrm{mbar}\right)$ pour générer les harmoniques d'ordres élevés. L'énergie IR résiduelle est éliminée à l'aide d'un filtre d'aluminium de $200 \mathrm{~nm}$ d'épaisseur. L'ensemble des dispersions introduites entre autre par les deux lames $\lambda / 4$ et le hublot d'entrée de la chambre de génération d'harmonique sont pré-compensées par un réglage du compresseur de la chaîne laser. Ceci permet d'assurer que les impulsions utilisées sont toutes proches de la limite de Fourier. Comme le montre la figure 2, un miroir concave troué permet de superposer spatialement les faisceaux harmonique (pompe) et infrarouge (sonde 9fs) dans une géométrie colinéaire.

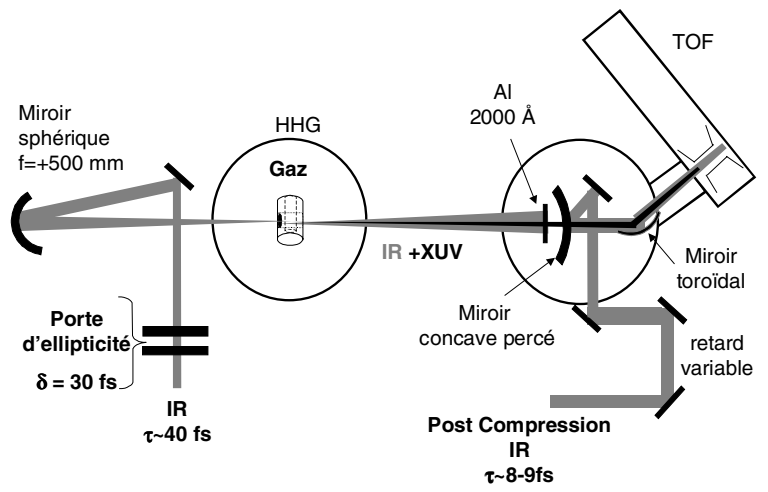

Figure 2. Dispositif expérimental du LLC. Mesure temporelle directe du confinement HHG par corrélation croisée XUV/IR ultra-bref.

La concavité du miroir a été choisie pour ajuster la divergence du faisceau IR et assurer un recouvrement des foyers IR et XUV. Les deux faisceaux sont ensuite focalisés par un miroir toroïdal dans la zone sensible d'un spectromètre à temps de vol (TOF) pour électrons, remplie d'argon. Les photo-électrons émis lors de l'ionisation à deux couleurs de l'argon sont alors détectés par des galettes 
micro-canaux et leur énergie est déduite du temps de vol. Une ligne à retard variable sur le premier bras (sonde IR) permet de faire varier le délai entre les impulsions XUV et IR avec une résolution temporelle inférieure au cycle optique.

\subsection{Résultats expérimentaux}

La figure (3.a) montre l'évolution d'un spectre de photo-électrons obtenu en fonction du délai XUV/IR. On voit apparaître, indépendamment du délai, des lignes continues qui correspondent à la photo-ionisation de l'argon par les harmoniques H13 à H25 (q étant l'ordre de l'harmonique Hq). Quand les impulsions pompe (XUV) et sonde (IR) se recouvrent, on voit apparaitre des structures entre les harmoniques. Ces «bandes latérales » correspondent à l'absorption d'un photon harmonique plus l'absorption ou l'émission d'un photon IR. En enregistrant l'amplitude de ces bandes latérales en fonction du délai XUV/IR, ont obtient des traces de corrélation croisée XUV/IR. On peut alors en déduire la durée de l'émission XUV pour différentes «portes d'ellipticité». La figure (3.b) présente les traces de corrélation croisée pour la bande latérale BL18 (BL18 apparaissant entre les harmoniques H17 et H19) lorsque l'on passe d'une configuration «porte large» à «porte étroite».

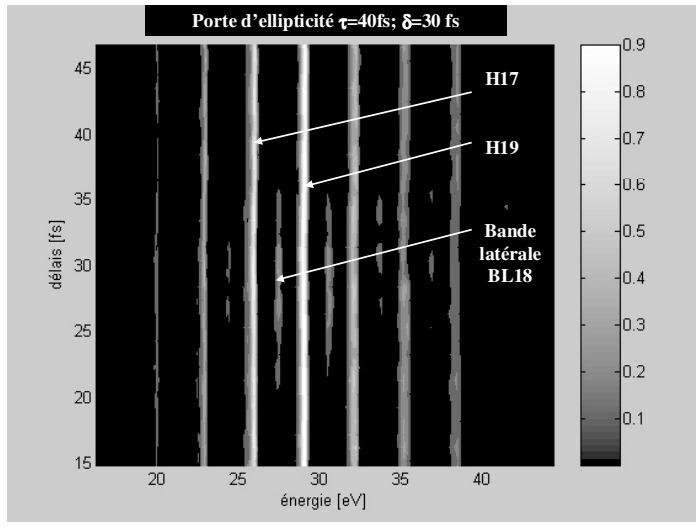

(a)

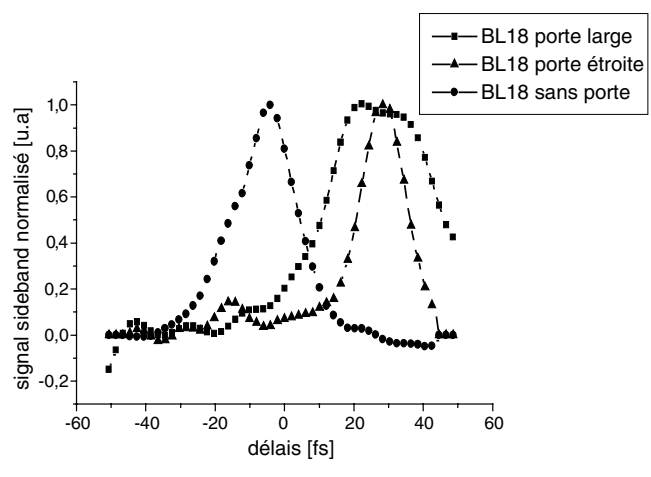

(b)

Figure 3. (a) Spectre de photo-électrons en fonction du délai XUV/IR (axe vertical). Les harmoniques H13 à H25 apparaissent à tous les délais, lorsque les deux impulsions sont superposées temporellement, les bandes latérales (BL) apparaissent entre les harmoniques.

(b) Traces de corrélation croisée de la bande latérale BL18 en configurations «porte étroite» (triangle), «sans porte» (cercle) et «porte large» (carré).

La durée moyenne $\tau_{\text {XUV }}$ des harmoniques H17 et H19 est ensuite déduite de ces traces de corrélation croisée par déconvolution de type gaussienne :

$$
\tau_{X U V}=\sqrt{\tau_{B L}^{2}-\tau_{I R}^{2}}
$$

où $\tau_{\mathrm{BL}}$ est la durée (FWHM) de la trace de corrélation croisée et $\tau_{I R}$ est la durée (FWHM) de la sonde IR. De cette façon nous obtenons une durée d'émission XUV de 34 fs en configuration «porte large», de 18 fs en configuration «sans porte» et de 11,5 fs en configuration «porte étroite» en accord avec un calcul de la durée de la porte d'ellipticité à 11,6 fs. Compte tenue de la durée de l'émission XUV en configurations «porte étroite» et «sans porte», cette technique nous permet donc d'observer que l'émission harmonique est bien confinée par une modulation temporelle de l'ellipticité fondamentale et que la durée de l'impulsion XUV en configuration «porte étroite» est alors comparable à un calcul de la durée de la porte d'ellipticité. 


\section{MESURE SPECTRALE DU CONFINEMENT HHG}

Des expériences précédentes ont permis de mettre en évidence une évolution spectrale de la largeur des harmoniques en fonction de la porte d'ellipticité [11, 12]. Ces résultats sont en accord avec un confinement de la HHG produite par des impulsions IR de $\sim 30$ fs dont l'ellipticité a été modulée. Nous avons récemment réalisé ces expériences en modulant cette fois l'ellipticité d'une impulsion IR plus courte $(9 \mathrm{fs})$ issue d'un système de post-compression.

\subsection{Dispositif expérimental}

Nous avons utilisé la ligne basse énergie du CELIA qui permet de disposer d'impulsions IR (805 nm) de 35 fs à une cadence de $1 \mathrm{kHz}$. Par une technique de «post compression» basée sur des capillaires $[9,10]$, nous disposons d'impulsions IR de $\tau=9$ fs et d'une énergie de $350 \mu \mathrm{J}$ en entrée de chambre de génération d'harmonique. Nous avons modulé l'ellipticité des impulsions de 9 fs qui sont ensuite utilisées pour produire les harmoniques. Le délai introduit par la lame $\lambda / 4$ d'ordre multiple est de $15 \mathrm{fs}$ ce qui ce traduit par une porte d'ellipticité de durée $1 \mathrm{fs}$ (en configuration «porte étroite»). L'impulsion est alors focalisée à l'aide d'un miroir sphérique dans une cellule d'argon de $15 \mathrm{~mm}$ de long $\left(\mathrm{P}_{\mathrm{Ar}}=\right.$ $30 \mathrm{mbar}$ ). Un film mince d'aluminium d'épaisseur $200 \mathrm{~nm}$ permet de d'éliminer sélectivement l'IR et les harmoniques d'ordres inférieurs à 11 . Le spectre harmonique est alors enregistré grâce à un spectromètre XUV constitué d'un miroir toroïdal, d'un réseau plan et d'un photomultiplicateur placé après une fente.

\subsection{Résultats expérimentaux}

Nous avons enregistré les spectres HHG pour les configurations «sans porte» et «porte étroite», comme le montre la figure 4.

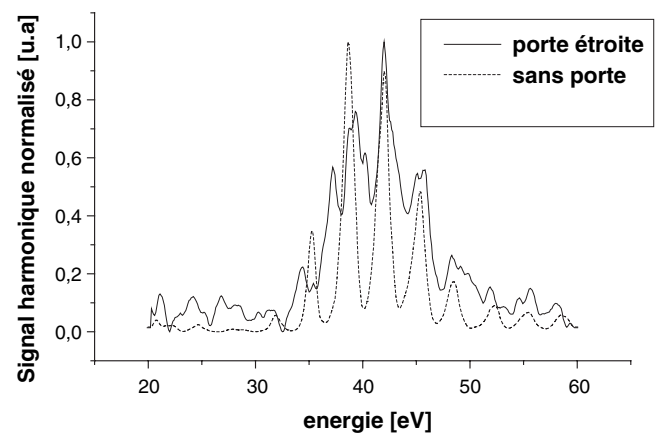

Figure 4. Spectre HHG obtenu dans l'argon avec une impulsion IR incidente de 9 fs dont l'ellipticité est modulée temporellement pour deux configurations de porte d'ellipticité. Configuration «sans porte» (courbe pointillée) et configuration «porte étroite» (courbe continue).

Nous constatons d'abord que l'amplitude des harmoniques basses $(\mathrm{q}<23)$ est relativement faible (environ $10 \%$ de l'amplitude maximale) quelle que soit la configuration de la porte. Compte tenue de la longueur de la cellule, nous attribuons cette faible transmission à la réabsorption des harmoniques basses par le milieu (la longueur de réabsorption de l'harmonique H19 étant de 5,5 mm dans l'argon à une pression de $\mathrm{P}_{\mathrm{Ar}}=30 \mathrm{mbar}$ ). Mais surtout, nous observons clairement un élargissement spectral des harmoniques vers un quasi continuum en configuration «porte étroite». Quelques structures harmoniques restent cependant apparentes. L'acquisition du spectre harmonique s'effectuant sur un grand nombre de tirs laser, l'aspect du spectre peut être interprété comme résultant de la moyenne entre un spectre continu correspondant à l'émission d'une impulsion XUV unique et d'un spectre harmonique discret 
correspondant à l'émission de deux impulsions attosecondes dans le train. Cette interprétation est cohérente avec la durée théorique de la porte d'ellipticité ( $\tau_{\text {gate }}=1 \mathrm{fs}$ ).

\subsection{Interprétation théorique}

Ce scénario est en accord avec une modélisation théorique de la génération d'harmonique lorsque la durée de confinement est proche du demi cycle optique IR. On atteint alors un régime limite où la phase absolue du champ IR influe de façon cruciale sur l'émission du train d'impulsions XUV [6]. Cette phase absolue $\varphi$ apparaît dans la description du champ laser $E(t)=E_{0}(t) \cos \left(\omega_{0} t+\varphi\right)$, où $E_{0}(t)$ correspond à l'enveloppe « lentement » variable et $\omega_{0}$ désigne la fréquence IR. Cette phase n'est pas stabilisée dans nos expériences ce qui se traduit par la détection d'un spectre moyenné sur toutes les phases absolues du champ IR. La modélisation de l'émission harmonique est ici dérivée du modèle semi-classique en trois étapes décrit plus haut. La théorie est généralisée pour une polarisation incidente arbitraire et est décrite dans la référence [6]. Un soin particulier a été apporté à la description de l'évolution du paquet d'onde électronique dans un champ de polarisation quelconque. Ainsi, le modèle prend en compte l'élargissement transverse et l'influence du champ Coulombien de l'ion parent sur l'évolution de la fonction d'onde électronique. De même, la probabilité d'ionisation dépendant de la polarisation a été calculée en résolvant l'équation de Schrödinger sur une base de B-spline [13] avec un modèle de potentiel atomique. La figure 5 illustre l'influence de la phase absolue sur le nombre de «trajectoires » du paquet d' onde électronique conduisant à l'émission d'impulsions XUV pendant la porte de polarisation.

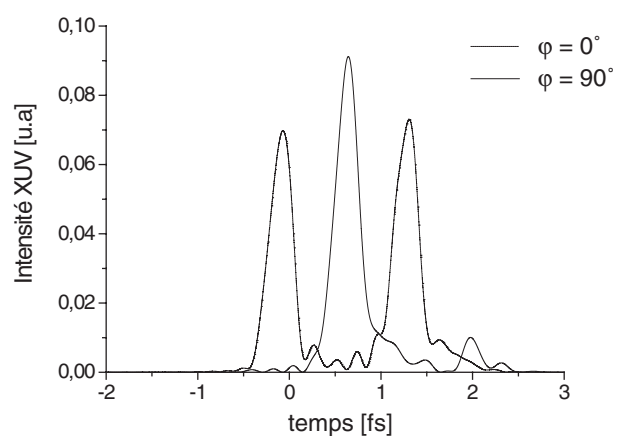

Figure 5. Profil temporel calculé en configuration «porte étroite» pour une impulsion incidente de $\tau=9$ fs et un délai de $\delta=15 \mathrm{fs}$ en considérant deux phases absolues du champ fondamental : $\varphi=0^{\circ}$ (ligne pointillée) et $\varphi=90^{\circ}$ (ligne continue).

Les profils temporels attosecondes sont calculés pour deux phases absolues du champ IR $\left(\varphi=0^{\circ}\right.$ et $\varphi=90^{\circ}$ ) dans le cas d'une porte de polarisation correspondant ( $\tau=9 \mathrm{fs}, \delta=15 \mathrm{fs}$ ) à nos conditions expérimentales. On voit alors qu'en fonction de cette phase absolue, on obtient soit une impulsion unique soit deux impulsions.

\section{PERSPECTIVES AU CELIA}

Afin d'isoler une seule impulsion attoseconde dans le train, une des possibilités consisterait à stabiliser la phase absolue du champ. Cette technique a déjà été mise en œuvre pour des impulsions IR de 5 fs qui ont permis de produire une impulsion XUV isolée de 250 as [7]. Cependant, cette technique a sans doute atteint la durée minimale accessible en raison de la sélection spectrale des harmoniques de la coupure requise. En revanche, notre technique ne nécessite pas de sélection spectrale et permet en principe de franchir la limite des 100 as. D'autre part, il est important de remarquer que la porte d'ellipticité offre une alternative permettant éventuellement de s'affranchir de la stabilisation de la phase absolue. En effet, il semble possible de ne produire qu'une seule impulsion attoseconde ou aucune en utilisant une porte de polarisation plus courte qu'un demi cycle optique. Cette configuration peut être 
réalisée en utilisant par exemple des impulsions de 6 fs et un délai de 6 fs introduit par une lame $\lambda / 4$ d'ordre multiple. La variation de phase absolue se traduit alors essentiellement par une diminution du taux de répétition effectif de la source XUV attoseconde. Le choix d'une impulsion plus courte avec un délai égal à sa durée $(\delta \sim \tau=6 \mathrm{fs}$ ) permet d'obtenir une intensité maximale au centre de la porte de polarisation favorisant en principe l'efficacité de génération d'une impulsion attoseconde unique. Enfin, notre méthode permet aisément de faire varier le nombre d'impulsions attosecondes et d'étudier continûment la transition vers l'émission d'une impulsion unique.

\section{CONCLUSION}

L'expérience réalisée au LLC nous a permis de mettre en évidence, par une mesure temporelle directe, un contrôle du confinement de l'émission harmonique compatible avec les prédictions théoriques. L'expérience et les calculs réalisés au CELIA indiquent qu'il est possible de générer une ou deux impulsions attosecondes dans le train en appliquant cette technique sur des impulsions ultra-brèves (9 fs). Nous envisageons de moduler l'ellipticité d'une impulsion IR plus courte ( $\sim 6 \mathrm{fs})$ afin de ne produire qu'une impulsion isolée sans nécessairement stabiliser la phase absolue du champ fondamental. Bénéficiant d'un large spectre harmonique émis lors de l'impulsion, nous espérons ainsi produire une impulsion isolée sub-100 as.

\section{Remerciements}

Nous remercions le réseau ATTO européen (HPRN-2000-001333) dans le cadre duquel la collaboration entre le CELIA et le LLC a eu lieu, la région Aquitaine ainsi que les Fonds Européen de Développement Régional (FEDER).

\section{Bibliographie}

[1] P. M. Paul, E. S. Toma, P. Breger, G. Mullot, F. Augé, Ph. Balcou, H. G. Muller, P. Agostini: Science 292, 1689 (2001).

[2] M. Hentschel, R. Kienberger, Ch. Spielmann, G. A. Reider, N. Milosevic, T. Brabec, P. Corkum, U. Heinzmann, M. Drescher, F. Krausz. : Nature 414, 509 (2001).

[3] P. B. Corkum, N. H. Burnett, M. Y. Ivanov: Opt. Lett. 19, 1870 (1994). M. Y. Ivanov, P. B. Corkum, T. Zuo, A. Bandrauk: Phys. Rev. Lett. 74, 2933 (1995).

[4] K. J. Schafer, B. Yang, L. F. Di Mauro, K. C. Kulander: Phys. Rev. Lett. 70, 1599 (1993).

[5] G. Farkas, C. Toth: Phys. Lett. A 168, 447 (1992).

S. E. Harris, J. J. Macklin, and T. W. Hinsh: Opt. Commun. 100, 487 (1993).

[6] V. Strelkov, A. Zaïr, O. Tcherbakoff, R. Lopez-Martens, E. Cormier, E. Mével, E. Constant: Appl. Phys. B 78, 879-884 (2004).

[7] R. Kienberger, E. Goulielmakis, M. Uiberacker, A. Baltuska, V. Yakovlev, F. Bammer, A. Scrinzi, Th. Westerwalbesloh, U. Kleineberg, U. Heinzmann, M. Drescher, F. Krausz. : Nature, 427 (6977) 817-21 (2004).

[8] R. Lopez-Martens, J. Mauritsson, P. Johnsson, A. L'Huillier and O. Tcherbakoff, A. Zaïr, E. Mével, E. Constant : PRA 69, 053811 (2004).

A. Zaïr, O. Tcherbakoff, E. Mével, E. Constant and R. Lopez-Martens, J. Mauritsson, P. Johnsson, A. L'Huillier : Appl. Phys. B 78, 869-872 (2004).

[9] M. Nisoli, S. De Silvestri, O. Svelto : Appl. Phys. Lett. 68, 2793 (1996).

[10] M. Nisoli, S. De Silvestri, O. Svelto: Opt. Lett. 22, 522 (1997).

[11] O. Tcherbakoff, E. Mével, D. Descamps, J. Plumridge, E. Constant : PRA 68, 43804 (2003).

[12] M. Kovacev, Y. Mairesse, E. Priori, H. Merdji, O. Tcherbakoff, P. Monchicourt, P. Breger, E. Mével, E. Constant, P. Salières, B. Carré, P. Agostini : Eur. Phys. J. D 26, 79 (2003).

[13] E. Cormier, P. Lambropoulos: J. Phys. B 30, 77 (1997). 